\title{
Self-Interacting Random Motions
}

\author{
Bálint Tóth
}

\begin{abstract}
We present a brief survey of results concerning self-interacting random walks and self-repelling continuous random motions. A self-interacting random walk (SIRW) is a nearest neighbour walk on the one-dimensional integer lattice $\mathbb{Z}$ which starts from the origin and at each step jumps to a neighbouring site, the probability of jumping along a bond being proportional to $w$ (number of previous jumps along that lattice bond), where $w: \mathbb{N} \rightarrow \mathbb{R}_{+}$is a monotone weight function. We consider various weight functions, the most natural and most interesting one being $w(n)=\exp \{-\beta n\}$, where $\beta>0$ is a fixed constant parameter. This weight function defines the so-called 'myopic self-repelling' random walk. Other weight functions are also considered. We present functional limit theorems for the local time processes of these random walks and limit theorems for the position of the random walker at late times, under anomalous scaling rate. A generalization of the Ray-Knight theory of local time is in the background of these results.

In the second part of this note we present results concerning the construction and primary properties of a continuous, locally self-repelling process $X_{t}$. The process is a.s. continuous and recurrent, it has a regular occupation time density (local time) denoted $L_{t}(x)$, and the self-repellence of its trajectories is achieved by the dynamical driving mechanism formally expressed as $d X_{t}=-\operatorname{grad} L_{t}\left(X_{t}\right) d t$. This means that the process $X_{t}$ is instantaneously pushed in the direction of the decrease of its local time. The constructed process is self-similar with scale-exponent $\nu=2 / 3$ and has non-trivial local variation of order $3 / 2$ (in contrast with the finite quadratic variation of semi-martingales).

This note is an abridged version of the survey paper [16]. Full proofs of the cited results can be found in [12]-[15] and [17].
\end{abstract}

\section{Self-Interacting Random Walks on $\mathbb{Z}$}

The need for investigation of late-time asymptotics of random walks with long memory arose naturally in the probability and statistical physics literature. See e.g. $[1,2,3,6,9,10]$ etc. and the survey chapters of [7] and [8] for the historical origins of the problems. We present a survey of results concerning the long time asymptotics of self-interacting random walks on $\mathbb{Z}$ defined as follows: the walk $X_{i}$ starts from the origin of the lattice and at time $i+1$ it jumps to one of the two neighbouring sites of $X_{i}$, so that the probability of jumping along a bond of the 
lattice is proportional to

$$
w \text { (number of previous jumps along that bond) }
$$

where

$$
w: \mathbb{N} \rightarrow \mathbb{R}_{+}
$$

is a weight function to be specified later. Complicated long-time memory effects are built up by the self-interaction mechanism driving these random walk. In papers [12]-[15] we developed a method which allowed us to prove limit theorems for the distributions of the late time position of a wide class of SIRWs.

\subsection{Setup and main examples}

Let $\mathbb{N} \ni i \mapsto X_{i} \in \mathbb{Z}$ be a nearest neighbour walk, starting from the origin of the lattice. I.e. $X_{0}=0$ and $\left|X_{i+1}-X_{i}\right|=1$ for any $i \in \mathbb{N}$. For such a walk we define the (edge) local time process in the most natural usual way:

$$
L_{i}(x):=\#\left\{j \in[0, i):\left(X_{j}, X_{j+1}\right)=(x, x+1) \text { or }(x+1, x)\right\} .
$$

That is: $L_{i}(x)$ is the number of jumps across the edge $\langle x, x+1\rangle$ in either direction, performed before time $i$.

Let $w: \mathbb{N} \rightarrow \mathbb{R}_{+}$be a weight function, either monotone non-decreasing or monotone non-increasing. The SIRW defined by the weight function $w(\cdot)$ is a nearest neighbour walk $X_{i}$ on $\mathbb{Z}$ with $X_{0}=0$ and governed by the law

$$
\begin{aligned}
\mathbf{P}\left(X_{i+1}=X_{i}+1 \mid \underline{X}_{0}^{i}\right) & =\frac{w\left(L_{i}\left(X_{i}\right)\right)}{w\left(L_{i}\left(X_{i}\right)\right)+w\left(L_{i}\left(X_{i}-1\right)\right)} \\
& =1-\mathbf{P}\left(X_{i+1}=X_{i}-1 \mid \underline{X}_{0}^{i}\right),
\end{aligned}
$$

where we used the shorthand notation $\underline{X}_{0}^{i}:=\left(X_{0}, X_{1}, \ldots, X_{i}\right)$. In plain words: the random walker jumps to one of the two nearest neighbour sites so that the probability of jumping across an edge of the lattice is always proportional to the weight associated to the number of previous jumps across that edge. It is intuitively clear that monotone non-increasing weight functions define self-repelling walks while monotone non-decreasing weight functions define self-attracting walks. It is also clear that in the conditional jump probabilities (1) the full past history of the walk plays an important role, so these walks have extremely long memory, they are strongly non-Markovian. So, one should expect interesting, non-trivial long time asymptotic behaviour.

It turns out that the asymptotic behaviour of the SIRW is very sensitive to the choice of the weight function $w(\cdot)$. The following classes of weight functions have been considered:

(1) In the exponentially self-repelling case

$$
w(n)=\exp (-\beta n), \quad \beta>0,
$$

the random walk scales like $n^{-2 / 3} X_{n}$ (see [12]). 
(2) The subexponentially self-repelling random walk governed by

$$
w(n)=\exp \left(-\beta n^{\kappa}\right), \quad \beta>0, \kappa \in(0,1)
$$

scales like $n^{-(\kappa+1) /(\kappa+2)} X_{n}($ see $[13])$.

(3) In case of power law self-interaction

$$
w(n)=\frac{1}{1-\alpha}\left(\frac{n}{2}\right)^{\alpha}-\frac{B}{(1-\alpha)^{2}}\left(\frac{n}{2}\right)^{\alpha-1}+\mathcal{O}\left(n^{\alpha-2}\right), \quad B \in \mathbb{R}, \alpha \in(-\infty, 1),
$$

We found three very different sub-cases (see [14]): If $\alpha \in(-\infty, 0)$ (polynomial self-repellence), then the correct scaling is $n^{-1 / 2} X_{n}$ but the limit law is not gaussian and does not depend essentially on the parameters. If $\alpha=0$ (asymptotic freedom), then the correct scaling is again $n^{-1 / 2} X_{n}$ but the limit law will depend essentially on the value of

$$
\delta:=2 w(0)^{-1}+2 \sum_{j=1}^{\infty}\left(w(2 j)^{-1}-w(2 j-1)^{-1}\right) .
$$

For related results on the so-called random walk and Brownian motion perturbed at extrema see also $[4,5,11,18]$ etc.

(4) Finally, if $\alpha \in(0,1)$ (weak reinforcement) then the walk scales like $n^{-(1-\alpha) /(2-\alpha)} X_{n}$ (see [15]).

\subsection{Limit theorems for the exponentially self-repelling random walk}

Pars pro toto we present in some detail the main results (limit theorems) concerning the 'physically' most interesting case, the so-called 'true self-avoiding' or exponentially self-repelling walk.

For a nearest neighbour random walk $\mathbb{N} \ni i \mapsto X_{i} \in \mathbb{Z}$ we define the upcrossing, downcrossing processes:

$$
\begin{aligned}
U_{i}(x) & :=\#\left\{j \in[0, i):\left(X_{j}, X_{j+1}\right)=(x, x+1)\right\}, \\
D_{i}(x) & :=\#\left\{j \in[0, i):\left(X_{j}, X_{j+1}\right)=(x+1, x)\right\}, \quad i \in \mathbb{N}, x \in \mathbb{Z} .
\end{aligned}
$$

Clearly $L_{i}(x):=U_{i}(x)+D_{i}(x)$. It is straightforward that for any $i \geq 0$ we have

$$
i=\sum_{y \in \mathbb{Z}} L_{i}(y)
$$

The inverse local time processes are

$$
T_{x, m}^{\mathrm{U}}:=\inf \left\{i \in \mathbb{N}: U_{i}(x) \geq m\right\}, \quad T_{x, m}^{\mathrm{D}}:=\inf \left\{i \in \mathbb{N}: D_{i}(x) \geq m\right\} .
$$

Finally, the local time processes stopped at inverse local times are

$$
\Lambda_{x, m}^{*}(y):=L_{T_{x, m}^{*}}(y),
$$

Hereafter the superscript $*$ stands for either $U$ or $D$. In $\Lambda_{x, m}^{*}(y)$ one should think about $x \in \mathbb{Z}$ and $m \in \mathbb{Z}_{+}$as fixed parameters and $y \in \mathbb{Z}$ variable. From (2) it 
follows that for any $x \in \mathbb{Z}$ and $m \in \mathbb{N}$

$$
T_{x, m}^{*}=\sum_{y \in \mathbb{Z}} \Lambda_{x, m}^{*}(y) .
$$

The quantities defined above make perfectly good sense for any nearest neighbour walk on $\mathbb{Z}$.

We also need the reflected-absorbed Brownian motion process. Fix the parameters $x \in \mathbb{R}$ and $h \in \mathbb{R}_{+}$and let $\mathbb{R} \ni y \mapsto W(y) \in \mathbb{R}$ be a two-sided standard Brownian motion with $W(0)=0$. Then

$$
\Lambda_{x, h}(y):=|W(y-x)+h| \mathbb{1}_{\left\{\omega_{x, h}^{-} \leq y \leq \omega_{x, h}^{+}\right\}}
$$

where

$$
\begin{aligned}
& \omega_{x, h}^{-}:=\sup \{y<\min \{x, 0\}:|W(y-x)+h|=0\}, \\
& \omega_{x, h}^{+}:=\inf \{y>\max \{x, 0\}:|W(y-x)+h|=0\} .
\end{aligned}
$$

In plain words: $y \mapsto \Lambda_{x, h}(y)$ is a two-sided Brownian motion starting at 'time' $y=x$ from level $\Lambda_{x, h}(x)=h$ which in the 'time' interval $[\min \{x, 0\}, \max \{x, 0\}]$ is reflected at level 0 and outside this interval is absorbed at first hitting of level 0 . The total area under the curve $y \mapsto \Lambda_{x, h}(y)$ is

$$
T_{x, h}:=\int_{-\infty}^{\infty} \Lambda_{x, h}(y) d y=\int_{\omega_{x, h}^{-}}^{\omega_{x, h}^{+}} \Lambda_{x, h}(y) d y .
$$

If $(x, h) \in \mathbb{R} \times \mathbb{R}_{+}$and $(x, h) \neq(0,0)$ the random variable $T_{x, h}$ has an absolutely continuous distribution. Its density is $\varrho(t, x, h)$ with Laplace transform (in the $t$ variable $\widehat{\varrho}(s, x, h)$ :

$$
\varrho(t, x, h):=\frac{\partial}{\partial t} \mathbf{P}\left(T_{x, h}<t\right), \quad \widehat{\varrho}(s, x, h):=s \int_{0}^{\infty} \varrho(t ; x, h) e^{-s t} d t .
$$

We shall also use

$$
\pi(t, x):=\int_{0}^{\infty} \varrho(t, x, h) d h, \quad \widehat{\pi}(s, x):=\int_{0}^{\infty} \widehat{\varrho}(s, x, h) d h .
$$

Scale invariance of Brownian motion implies:

$$
\begin{array}{ll}
\alpha \varrho\left(\alpha t, \alpha^{2 / 3} x, \alpha^{1 / 3} h\right)=\varrho(t, x, h), & \alpha^{2 / 3} \pi\left(\alpha t, \alpha^{2 / 3} x\right)=\pi(t, x), \\
\alpha \widehat{\varrho}\left(\alpha^{-1} s, \alpha^{2 / 3} x, \alpha^{1 / 3} h\right)=\widehat{\varrho}(s, x, h), & \alpha^{2 / 3} \widehat{\pi}\left(\alpha^{-1} s, \alpha^{2 / 3} x\right)=\widehat{\pi}(s, x) .
\end{array}
$$

Theorem 1.1. Given $t \in(0, \infty)$ (respectively, $s \in(0, \infty)$ ) fixed, $(x, h) \mapsto \varrho(t, x, h)$ (respectively, $(x, h) \mapsto \widehat{\varrho}(s, x, h))$ is a probability density on $\mathbb{R} \times \mathbb{R}_{+}$. That is:

$$
\int_{-\infty}^{\infty} \pi(t, x) d x=1=\int_{-\infty}^{\infty} \widehat{\pi}(s, x) d x
$$

The following Ray-Knight-type invariance principle for the local time process (stopped at inverse local times) and its corollary are the clue to the asymptotic description of the self-interacting walk: 
Theorem 1.2. Let $x \in \mathbb{R}, h \in \mathbb{R}_{+}$be fixed and the superscript $*$ stand for either $U$ (upcrossing) or $D$ (downcrossing). Then, as $n \rightarrow \infty$,

$$
(\sqrt{n} \sigma)^{-1} \Lambda_{[n x],[\sqrt{n} \sigma h]}^{*}([n y]) \Rightarrow \Lambda_{x, h}(y),
$$

in the function space $D(-\infty, \infty)$ endowed with the Skorohod topology.

Using the identity (3) we get the following:

Corollary 1.3. Under the same conditions

$$
\left(n^{3 / 2} \sigma\right)^{-1} T_{[n x],[\sqrt{n} \sigma h]}^{*} \Rightarrow T_{x, h},
$$

Let $s>0$ be fixed and $\theta_{n}$ a geometrically distributed stopping time which is independent of the walk $X_{i}$,

$$
\mathbf{P}\left(\theta_{N}=k\right)=\left(1-e^{-s / n}\right) e^{-s k / n} .
$$

The next statement is a limit theorem for the distribution of the location of the walk $X_{i}$, stopped at the random stopping time $\theta_{n}$, with $n \rightarrow \infty$. It follows from the conversion of the full information contained in the previous corollary.

Theorem 1.4. Let $s>0$ and $x \in \mathbb{R}$ be fixed and $\theta_{n}$ a geometric stopping time, independent of the walk $X_{i}$, distributed according to (4). Then, as $n \rightarrow \infty$,

$$
\mathbf{P}\left(n^{-2 / 3} X_{\theta_{n}}<x\right) \rightarrow \int_{-\infty}^{x} \widehat{\pi}(\sigma s, y) d y
$$

Remarks 1.5. (1) In the original paper a slightly stronger statement was proved: the local version of this limit theorem, i.e. pointwise convergence of the properly defined density functions, rather than convergence of the distribution functions.

(2) The statement in theorem1.4 is a little bit short of stating the limit theorem for deterministic time:

$$
\mathbf{P}\left(n^{-2 / 3} X_{[n t]}<x\right) \rightarrow \int_{-\infty}^{x} \pi\left(\sigma^{-1} t, y\right) d y,
$$

In order to convert (5) to (6) some refined Tauberian argument would be needed, which we were not able to push through. But, of course, we can conclude that, if $X_{[n t]}$ obeys any limit law as $n \rightarrow \infty$, then (6) also must hold.

\section{The True Self-Repelling Motion}

\subsection{Setup}

In the previous section a limit theorem was stated for the one dimensional marginal distributions of $X_{t}^{(n)}:=n^{-2 / 3} X_{[n t]}$, where $X_{j}$ was the exponentially self-repelling (or myopic self-avoiding) random walk on $\mathbb{Z}$, defined by the weight function $w(n)=$ $\exp (-\beta n)$. Now we consider the problem of invariance principle, i.e. that of the weak convergence of the process $X_{t}^{(n)}$, as $n \rightarrow \infty$. The results presented in this section are quoted from [17], where we constructed the presumed limit-process: 
a robust, self-similar stochastic process $\mathbb{R}_{+} \ni t \mapsto X_{t} \in \mathbb{R}$, with all the natural properties requested from a locally self-repelling continuous motion.

The following are the fundamental properties of the true self-repelling motion $X_{t}$ constructed in [17]:

Continuity, recurrence: Almost surely, $X_{0}=0$, the process $t \mapsto X_{t}$ is continuous on $[0, \infty)$ and for any $x \in \mathbb{R}$, the set of times $\left\{t \geq 0: X_{t}=x\right\}$ is unbounded.

Scaling: For all $\alpha>0,\left(X_{\alpha t}, t \geq 0\right)$ and $\left(\alpha^{2 / 3} X_{t}, t \geq 0\right)$ are identical in law.

Local variation: For all $\varepsilon>0$, define by induction $\theta_{0}^{\varepsilon}:=0$ and for all $n \geq 1$,

$$
\theta_{n}^{\varepsilon}:=\inf \left\{t>\theta_{n-1}^{\varepsilon}:\left|X_{t}-X_{\theta_{n-1}^{\varepsilon}}\right|=\varepsilon\right\} .
$$

Then, for all $t \geq 0$,

$$
\mathrm{P}-\lim _{\varepsilon \downarrow 0} \varepsilon^{3 / 2} \sup \left\{n \geq 0: \theta_{n}^{\varepsilon} \leq t\right\}=\frac{2}{\sqrt{\pi}} t .
$$

(Here and in the sequel $\mathrm{P}$ - lim stands for limit in probability.)

Occupation-time density: Almost surely, for all $t \geq 0$, the occupation-time measure of $X_{s}$ on the time-interval $[0, t]$ has a bounded density with respect to the Lebesgue measure and this density has a continuous version that we denote by $L_{t}(\cdot)$. We call $L_{t}(x)$ the local time of $X$ at time $t$ and position $x$.

Markov property of $\left(\boldsymbol{X}_{\boldsymbol{t}}, \boldsymbol{\mu}_{\boldsymbol{t}}\right)$ : The process $\left(X_{t}, L_{t}(\cdot)\right)_{t \geq 0}$, or equivalently the process $\left(X_{t}, \mu_{t}\right)_{t \geq 0}$, is a Markov process.

Locality: The self-interaction is local in the following sense: For all $t \geq 0$, the law of $X$ just after $t$ depends only on $L_{t}$ restricted to the immediate neighbourhood of the point $X_{t}$. In other words, the process $X_{t}$ is 'feeling' only the self-interaction due to the germ of its own past occupation-time measure at the points it is currently visiting.

The following property is of crucial importance: it describes in proper mathematical terms the phenomenon of local self-repellence.

Dynamical driving mechanism: There exists a random set $I \subset \mathbb{R}_{+}$, which is a.s. of full Lebesgue measure, such that for any $T \in I$

$$
\begin{aligned}
& \mathrm{P}-\lim _{\varepsilon \downarrow 0} \int_{0}^{T} \frac{L_{s}\left(X_{s}+\varepsilon\right)-L_{s}\left(X_{s}-\varepsilon\right)}{2 \varepsilon} d s= \\
&-X_{T}+\frac{1}{4}\left(\sup _{0 \leq s \leq T} X_{s}+\inf _{0 \leq s \leq T} X_{s}\right) .
\end{aligned}
$$

Unfortunately, we could prove (7) only for the random set of (stopping) times $I \subset \mathbb{R}_{+}$. Actually, this property should hold for all $T \in \mathbb{R}_{+}$. Phenomenologically, this equation states that the motion is driven by the negative gradient of the local time at the actual position, as long as the moving point is in the interior of the range swept in the past. This behaviour entitles us to call this process 'truely self-repelling'. In addition, at the edges of this range an instantaneous 
partial reflection (moving boundary condition) is felt. Indeed: writing (7) formally in differential form we find:

$$
d X_{t}=-\frac{\partial L_{t}\left(X_{t}\right)}{\partial x} d t+\left(\text { boundary effects at } \sup _{0 \leq s \leq t} X_{s} \text { and } \inf _{0 \leq s \leq t} X_{s}\right) .
$$

Strictly speaking, (8) does not make sense mathematically: the local time process is so singular that a 'differential equation' involving its gradient can not be rigorously defined $\left(L_{t}(\cdot)\right.$ has the same regularity properties as Brownian motion). Nevertheless, this formal way of writing may help the intuition about the dynamics of the process. Note, that there is no 'external noice', or 'external source of randomness' in the driving mechanism. One could think naively that such a mechanism would give rise to a deterministic motion. This is not the case: due to the extremely high singularity of this "differential equation", (8) has only truely stochastic solutions.

One of the main novelties of the process $X_{t}$ is exactly the fact that it is in striking contrast with our traditional intuition about a random motion being driven by local drift and external noice.

The full proof of the results can be found in [17]. The construction and derivation of key features of the process relies essentially on the construction and analysis of system of independent coalescing Brownian paths, emerging from every point of a two-dimensional space-time.

In the next subsection we present a not fully rigorous, phenomenological derivation of the driving mechanism. For the technical parts of the construction and analysis see the original publication.

\subsection{Phenomenological derivation of the dynamical driving mechanism.}

In [12] a limit theorem was proved, essentially for the distribution of $n^{-2 / 3} X_{n}$ as $n \uparrow \infty$, but the natural question of the asymptotics of the process

$$
X_{t}^{(N)}:=N^{-2 / 3} X_{[N t]}, \quad t \in \mathbb{R}_{+}
$$

in the limit $N \uparrow \infty$ remained open. In the following paragraphs we argue that, if the sequence of processes $t \mapsto X_{t}^{(N)}$ converges in distribution to a process $t \mapsto X_{t}^{(\infty)}$, as $N \uparrow \infty$, then the limit process is driven by the gradient of its local time, as claimed in (8). The forthcoming argument is based on a somewhat formal computation and it is by no means mathematically rigorous, but it sheds light on the essential phenomenon of local self-repellence.

Beside the scaled position process $t \mapsto X_{t}^{(N)}$ defined in (9) we define the properly scaled local time process of the exponentially self-repelling walk

$$
L_{t}^{(N)}(x):=N^{-1 / 3} L_{[N t]}\left(\left[N^{2 / 3} x\right]\right), \quad t \in \mathbb{R}_{+}, \quad x \in \mathbb{R}
$$

and we assume that the sequences of processes $X_{t}^{(N)}$ and $L_{t}^{(N)}(x)$ converge jointly weakly (in some vague topological space):

$$
\left(X_{t}^{(N)}, L_{t}^{(N)}(x)\right) \Rightarrow\left(X_{t}^{(\infty)}, L_{t}^{(\infty)}(x)\right)
$$


where $(t, x) \mapsto L_{t}^{(\infty)}(x)$ is assumed to be the local time of the process $t \mapsto X_{t}^{(\infty)}$. Let $\mathcal{F}_{n}$ be the $\sigma$-algebra generated by $\left(X_{0}, \ldots, X_{n}\right)$, then

$$
\begin{aligned}
\mathbf{E}\left(X_{n+1}-X_{n} \mid \mathcal{F}_{n}\right) & =-\tanh \left(\beta\left(L_{n}\left(X_{n}\right)-L_{n}\left(X_{n}-1\right)\right)\right) \\
\operatorname{Var}\left(X_{n+1}-X_{n} \mid \mathcal{F}_{n}\right) & =\cosh ^{-2}\left(\beta\left(L_{n}\left(X_{n}\right)-L_{n}\left(X_{n}-1\right)\right)\right) .
\end{aligned}
$$

So:

$$
X_{n}+\sum_{k=0}^{n-1} \tanh \left(\beta\left(L_{k}\left(X_{k}\right)-L_{k}\left(X_{k}-1\right)\right)\right)=: M_{n}
$$

is a martingale with quadratic variation process

$$
\langle M\rangle_{n}=\sum_{k=0}^{n-1} \cosh ^{-2}\left(\beta\left(L_{k}\left(X_{k}\right)-L_{k}\left(X_{k}-1\right)\right)\right)<n .
$$

Our object of study is the scaled form of (11):

$$
N^{-2 / 3} X_{[N t]}+N^{-2 / 3} \sum_{k=0}^{[N t]-1} \tanh \left(\beta\left(L_{k}\left(X_{k}\right)-L_{k}\left(X_{k}-1\right)\right)\right)=N^{-2 / 3} M_{[N t]} .
$$

The first term on the left-hand side of (13) is just $X_{t}^{(N)}$. From (12) in particular it follows that for any $T<\infty$

$$
\mathrm{P}-\lim _{N \uparrow \infty}\left(\sup _{0 \leq t \leq T}\left|N^{-2 / 3} M_{[N t]}\right|\right)=0
$$

so that the right hand-side of (13) is asymptotically negligible. A formal computation of the second term on the left-hand side of (13) follows: the first two steps are straightforward transformations using the definitions (9) and (10) of the scaled process and scaled local time:

$$
\begin{aligned}
& N^{-2 / 3} \sum_{k=0}^{[N t]-1} \tanh \left(\beta\left(L_{k}\left(X_{k}\right)-L_{k}\left(X_{k}-1\right)\right)\right) \\
= & N^{-1} \sum_{k=0}^{[N t]-1} N^{1 / 3} \tanh \left(\beta\left(L_{N k / N}\left(N^{2 / 3} X_{k / N}^{(N)}\right)-L_{N k / N}\left(N^{2 / 3} X_{k / N}^{(N)}-1\right)\right)\right) \\
= & N^{-1} \sum_{k=0}^{[N t]-1} N^{1 / 3} \tanh \left(\beta N^{1 / 3}\left(L_{k / N}^{(N)}\left(X_{k / N}^{(N)}\right)-L_{k / N}^{(N)}\left(X_{k / N}^{(N)}-N^{-2 / 3}\right)\right)\right) .
\end{aligned}
$$

The next step is the formal, non-rigorous one: we treat formally $L_{t}^{(N)}(x)$ as a smooth function and replace

$$
L_{t}^{(N)}(x)-L_{t}^{(N)}(x-\delta x) \quad \text { by } \quad \frac{\partial L_{t}^{(N)}(x)}{\partial x} \delta x
$$


to get

$$
\begin{gathered}
N^{-2 / 3} \sum_{k=0}^{[N t]-1} \tanh \left(\beta\left(L_{k}\left(X_{k}\right)-L_{k}\left(X_{k}-1\right)\right)\right) \\
"=" N^{-1} \sum_{k=0}^{[N t]-1} N^{1 / 3} \tanh \left(\beta N^{1 / 3} N^{-2 / 3} \frac{\partial L_{k / N}^{(N)}\left(X_{k / N}^{(N)}\right)}{\partial x}\right) \\
"=" \beta N^{-1} \sum_{k=0}^{[N t]-1} \frac{\partial L_{k / N}^{(N)}\left(X_{k / N}^{(N)}\right)}{\partial x}+\mathcal{O}\left(N^{-1 / 3}\right) \\
\Rightarrow " \beta \int_{0}^{t} \frac{\partial L_{s}^{(\infty)}\left(X_{s}^{(\infty)}\right)}{\partial x} d s .
\end{gathered}
$$

With the quotation marks "..." we intend to emphasize that these last equalities and convergence need more careful consideration. From (13), (14) and (15) we get

$$
X_{t}^{(\infty)}+\text { const. } \int_{0}^{t} \frac{\partial L_{s}^{(\infty)}\left(X_{s}^{(\infty)}\right)}{\partial x} d s=0
$$

which is indeed somewhat reminiscent of (7). The effect of 'pushing the boundaries of the range' and the right constant in front of the gradient term can not be recovered on this level of formal computations. We repeat again: this computation is nothing like rigorous, but on the phenomenological level it is convincing.

The same reasoning (on the same level of 'rigour') can be applied to the 'polymer model' proposed by Durrett and Rogers in [6]:

$$
X_{t}=B_{t}+\int_{0}^{t}\left\{\int_{0}^{s} f\left(X_{s}-X_{u}\right) d u\right\} d s
$$

where $f: \mathbb{R} \rightarrow \mathbb{R}$ is a smooth function of compact support and satisfies $f(-x)=$ $-f(x)$ and $\operatorname{sgn}(f(x))=\operatorname{sgn}(x)$. Defining $X_{t}^{(N)}=N^{-2 / 3} X_{N t}$, in the limit $N \rightarrow \infty$ $f$ transforms into $\delta^{\prime}$ and the same dynamical driving mechanism is found.

\section{Acknowledgements}

This work was partially supported by the following grants: OTKA T 26176 (National Fund for Scientific Research), FKFP 0638/1999 (Ministry of Culture and Education), TKI 'Stochastics@TUB' (Hungarian Academy of Sciences).

\section{References}

[1] D. Amit, G. Parisi and L. Peliti, Asymptotic behaviour of the 'true' self-avoiding walk, Phys. Rev. B, 27 (1983), 1635-1645.

[2] D. Coppersmith and P. Diaconis, Random walks with reinforcements, Stanford Univ. Preprint(1987) 
[3] B. Davis, Reinforced random walk, Probab. Theory Relat. Fields, 84 (1990), 203-229.

[4] B. Davis, Weak limits of preturbed random walks and the equation $Y_{t}=B_{t}+$ $\alpha \sup _{s \leq t} Y_{s}+\beta \inf _{s \leq t} Y_{s}$, Ann. Probab., 24 (1997), 2007-2023.

[5] B. Davis, Brownian motion and random walk perturbed at extrema, Probab. Theory Relat. Fields, 113 (1999), 501-518.

[6] R. T. Durrett and L. C. G. Rogers, Asymptotic behaviour of a Brownian polymer, Probab. Theory Relat. Fields,92 (1992), 337-349.

[7] G. Lawler, Intersections of Random Walks, Birkhäuser, Boston-Basel-Berlin, 1991.

[8] N. Madras and G. Slade, The Self-Avoiding Walk, Birkhäuser, Boston-Basel-Berlin, 1993.

[9] S. P. Obukhov and L. Peliti, Renormalisation of the "true" self-avoiding walk, J. Phys. A, 16 (1983), L147-L151.

[10] L. Peliti and L. Pietronero, Random walks with memory, Riv. Nuovo Cimento, 10 (1987), 1-33.

[11] M. Perman and W. Werner, Perturbed Brownian motions, Probab. Theory Relat. Fields, 108 (1997), 357-383.

[12] B. Tóth, The 'true' self-avoiding walk with bond repulsion on $\mathbb{Z}$ : limit theorems, Ann. Probab., 23 (1995), 1523-1556.

[13] B. Tóth, 'True' self-avoiding walk with generalized bond repulsion on $\mathbb{Z}$, J. Statist. Phys., 77 (1994), 17-33.

[14] B. Tóth, Generalized Ray-Knight theory and limit theorems for self-interacting random walks on $\mathbb{Z}$, Ann. Probab., 24 (1996), 1324-1367.

[15] B. Tóth, Limit theorems for weakly reinforced random walks, Studia Sci. Math. Hungar., 33 (1997), 321-337.

[16] B. Tóth, Self-interacting random motions - a survey, In: P. Révész, B. Tóth (eds): Random Walks, Bolyai Society Mathematical Studies, 9 (1999), 349-384.

[17] B. Tóth and W. Werner, The true self-repelling motion, Probab. Theory Relat. Fields, 111 (1998), 375-452.

[18] W. Werner, Some remarks on perturbed reflecting Brownian motion, Séminaire de Probabilités XXIX., Lecture Notes in Mathematics 1613, 37-43, Springer, 1995.

Institute of Mathematics,

Technical University Budapest,

H-1111 Budapest, Hungary

E-mail address: balint@math.bme.hu 